


\section{E M B E R S A N D S U B S C R I B E R S}

The Instituto Internacional de Literatura Iberoamericana was organized in 1938 in order to advance the study of Iberoamerican Literature and to promote cultural relations among the peoples of the Americas.

To this end, the Institute publishes the Revista Iberoamericana quarterly and sponsors the publication of noteworthy books by Iberoamerican authors -in their original language and in English translation-, and of learned works and textbooks.

Members of the Institute meet in Congresses every two years and are of two types: regular members who pay $\$ 12.00$ a year, except in Iberoamérica where the fee is $\$ 3.00$, and Patron Members who pay $\$ 15.00$ or more a year.

Institutions such as universities, colleges, and libraries may become subscribers (at $\$ 10.00$ a year or $\$ 3.00$ a year in lberoamérica), or $S u b$. scribing Patrons (at a minimum of $\$ 15.00$ a year) without holding mombership in the Institute.

Regular members and subscribers receive the forthcoming issues of the Revista Iberoamericana free, but Patrons (whether Members or Subscribers) receive, in addition, the forthcoming issues of all the publications of the Institute, and the Proceeding of the Congresses, etc. and their names will appear in the Revista Iberoamericana at the end of the year.

\section{SUBSCRIPTION APPLICATION}

I would like to become a subscriber to the Revista lberoamericana. Would you please send my subscription to the address indicated below. I understand that I may become a member by following the steps indicated in the statues of the Instituto, which may be obtained upon tequest.

$$
\begin{array}{rll}
\text { I am enclosing: } \$ 10.00 & \text { ( ) Regular subscription } \\
12.00 & \text { ( ) Regular Membet* } \\
3.00 & \text { ( ) Latin American Countries } \\
15.00 & \text { ( ) Patron subscription (all } \\
& & \text { publications) }
\end{array}
$$

Name

Address

City

Please make your checks payable to the Instituto Internacional de Literatura Iberoamericana and mail your dues to MRS. GLORIA J. Hardy, 657 AIR Bldg. University of Pittsburgh, Pittsburgh 13, Pennsylvania, U.S.A. All communications regarding the circulation and distribution of the publications of the Institute should also be addressed to Mrs. Hardy.

* In order to become a member, please send your curriculum vitae and the names of two members of the Institute who may recommend you. 


\section{SOCIOS Y SUSCRIPTORES}

El instituto internacional de Literatura iberoamericana se
organizó en 1938 con el fin de incrementar el estudio de la Literatura Iberoamericana e intensificar las relaciones culturales entre todos los pueblos de América.

Con este fin. el Instituto publica la Revista lberoamericana, por lo menos tres veces al año, y patrocina la publicación de obras notables de autores iberoamericanos - en el idioma original y en traducción inglesa-, y la de obras de enudición $y$ textos de enseñanza.

Los socios del Instituto se reúnen en Congreso cada dos años, y son de dos categorías: el socio de número, cuya cuota anual es de doce dólares, excepto en Ibetoamérica, donde es de sólo tres dólares, y el Socio Protector, cuya cuota es de quince dólares o más al año.

Las bibliotecas, colegios, universidades y demás instituciones que, sin ser socios, sí favorecen al Instituto, son de dos categorías: el suscriptor corriente, cuya cuota anual es de diez dólares y de sólo tres dólares en los países de Iberoamérica, y el Suscriptor Protector, cuya cuota es de doce dollares al año.

I.a Revisla theroamericana se envia a los socios de númcro y a los suscriptores corrientes del Instituto, y tanto los Socios Protectores como los Suscrtptores Protectores reciben, además de la Revista, las otras publicaciones que vayan saliendo, y Memorias. Los nombres de los Protectores se publican en la Revista lberoamericana al fin de cada año.

\section{N VITACION}

El Instituto invita cordialmente a quienes simpaticen con los fines que persigue, a que se hagan, ora socios, ora Protectores de él. Quienes asi lo apoyen deben enviar su cuota anual, por adelantado, en forma de giro postal o bancario pagadero al Instituto Internacional de Literatura Iberoamericana $y$ por conducto de la señora Gloria Hardy -657 AIR Bld., University of Pittsburgh, Pittsburgh 13, Pennsylvania, U. S. A.- que es la única persona encargada de la circulación y la distribución de las publicaciones del Instisuto.

\section{CUATRO NUMEROS POR AÑO}

A partit de 1970 la Revista lberoamericana se publica cuatro veces al año, en vez de dos y tres, como se habia hecho hasta entonces.

Lus manuscritos deberan ser enviados al Director, en original y una copia, antes de Jas siguientes fechas: 12 de noviembre, para el primer número (eneromarzo); 18 de enero, para el segundo número (abril-junio), $1^{9}$ de abril, para el tercer número (julio-septiembre) y $1^{9}$ de septiembre, para el cuarto número (octubte-diciembre). 


\title{
Revista Iberoamericana \\ Organo del Instituto Internacional \\ de Literatura Iberoamericana
}

Vol. XXXIX Julio-Diciembre de 1973 Núms. 84-85

PATROCINADA POR LA UNIVERSIDAD DE PITTSBURGH

\section{Director}

Alfredo A. Roggiano, 660 AIR Bldg, Universidad de Pittsburgh, Pittsburgh 13, Pennsylvania, U.S.A.

Comité Editorial (1973-1975)

Roberto González Echevarría, Cornell University

Héictor Martins, Indiana University

Emir Rodríguez Monegal, Yale University

Guillermo Sucre, University of Pittsburgh

\author{
SECRETARIO-Tesorero \\ Julio Matas, 658 AIR Bldg., University of Pittsburgh, Pittsburgh 13, \\ Pennsplvania, U.S.A. \\ JEFE DE CANJB
}

LILLAN S. DE LOZANo 


\title{
INSTITUTO INTERNACIONAL DE \\ LITERATURA IBEROAMERICANA
}

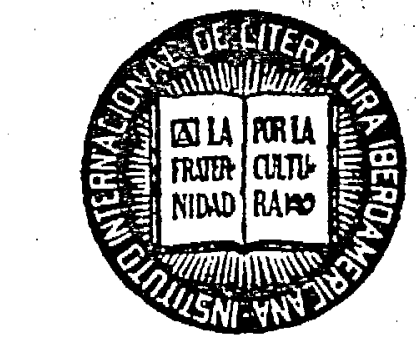

\author{
MESA DIRECTIVA \\ (1973-1975)
}

\section{PRESIDENTE}

Peter Earle University of Pennsylvania.

\section{VICEPRESIDEN'TES}

Donald Yates, Michigan State University

Luis Sáinz de Medrano, Universidad de Madrid

\section{VOCALES}

Angela Dellepiane, City College of the University City of New York Seymour Menton, University of California Irvine

SECRETARIO-TESORERO EJECUTIVO

Julio Matas, University of Pittsburgh

DIRECTOR DE PUBLICACIONES

Alfredo Roggiano, University of Pittsburgh 


\section{REVISTA IBEROAMERICANA}

PROPOSITOS

Esta REVISTA aspira a constituir, gradualmente, una vital representación de los valores espirituales de la creciente cultura iberoamericana.

Su director y asesores quieren hacer vivo el lema que cifra el ideal adoptado por nuestro Instituto: A LA FRATERNIDAd POR LA CULTURA.

Reflejará en sus páginas una clara imagen de la literatura y del pensamiento de Iberoamérica.

\section{NORMAS EDITORIALES}

La REVISTA IBEROAMERICANA sólo publicará artículos aceptados por su Director, quien será asesorado por la Comisión Editorial "Ad-hoc". Las ideas contenidas en los artículos que se publiquen pertenecen al autor, quien será único responsable de las mismas.

Se recomienda que en los manuscritos de artículos, notas y reseñas presentados para su publicación se sigan las normas de "The MLA Style Sheet" publicado en PMLA, lxvi (1951).

Los manuscritos deben ser enviados al Director, en original y copia. En caso de no ser aceptados para su publicación, sólo se devolverá el original si el autor envía el sobre con el correspondiente importe en estampillas o sellos de correos de U.S.A.

La reproducción de cualquier trabajo publicado en la R.I. deberá ser autorizado por el Director.

\section{CANJE Y SUSCRIPCIONES}

Todo lo referente a CANJE y demás intercambio de publicaciones con casas editoras, instituciones o autores deberá hacerse por intermedio del Jefe de Canje, y a tal efecto se ruega dirigirse a: LILlIAN S. LOzANo, 660 AIR Bldg., University of Pittsburgh, Pittsburgh 13, Pennsylvania, U. S. A. Todo lo referente a sUSCRIP. CIONEs, compras, órdenes de pago, etc., en que sea menester la intervención de la Tesorería, deberá hacerse por intermedio de la Secretaria-Tesorería-657 AIR Bldg., y a tal efecto se ruega escribir a: Mrs. Gloria Hardy, University of Pittsburgh, Pittsburgh 13, Pennsylvania, USA. 

Main Library R-039

University of Alabama

University, Alabama 35486

Abilene Christian College

Library R.514

Attn: Miss Collie F. Milliken

Abilene, Texas 79601

${ }^{*}$ Mr. \& Mrs. A. Owen Aldridge

2054 Foreign Langs. BIdg.

University of Illinois

Urbana, Illinois 61801

Dr. Humberto Alvarez

Prof. of Span. \& Latin Amer. Stud

Northeast Missouri State College

Kirksville, Missouri 63501

- Prof. Alberto Andino

1828 S. Dollison Av.

Springfield, Mo. 65804

* Shirley Arora

Spanish Department

University of Calif., L. A.

Los Angeles, CaliE. 90024

* Robert M. Assardo

Dept. of Foreign Langs.

University of Wisconsin

Stevens, Point, Wis. 54481

*Prof, J. B. Avalle-Arce

Euscaletxea

3904 Garrett Rd.

Durham, N. C. 27707

*Assistant Prof. Jorge Ayora

Dept. of Romance Langs.

University of Oregon

Eugene, Oregon 97403

* John Barta

Dept. of Foreign Langs.

Calif. State Univ.

Fresno, Calif. 93710

-Prof. Rubén Benitez

2809 S. Bentley

Los Angeles, Calif. 90063

*Prof. Rodney V. Bodden

Dept. of Romance Languages

University of Washington

Seattle, Washington 9810 S
Florence Bonhard

83 Fremont Place

Los Angeles, California 90005

"Wayne S. Bowen

Dept. of Foreign Langs.

Fresno State College

Fresno, California 93710

*Mr. Michael G. Brenner

10037 El Pinar Drive

Concord. Tenn. 37720

* Prof. J. S. Brushwood

Dept. of Spanish \& Port.

University of Kansas

Lawrence, Kansas 66044

California Baptist College

Annie Gabriel Libraty

8432 Magnolia Ave.

Riverside, Calif.

Serials Dept.

Univ. Research Library

Univ, of Calif.

Los Angeles, Calif. 90024

Cardinal Hayes

Library

Manhattan College

Bronx, New York 10471

*Dr. Clyde Hull Cantrell

P. O. Box 290

Auburn, Alabama 36830

* Prof, Carlos Cano

Dept. of Modern Langs.

University of S. Florida

Tampa, Florida 33620

*Dr. E. Dale Carter, Jr. 17220 Willard Street

Van Nuys, Calif. 91406

*Homero Castillo

P. O. Box 5579

Metropolitan Station

Los Angeles, Calif. 90055

* Rebeca Catz

300 El Camino Drive

Beverly Hills, Calif. 90212

*Prof. E. Chang-Rodríguez

Dept. of Romance Langs.

Queens College-City University of New York

Flushing, New York 11367
*Dr. Anthony Julio Ciccone

27 East Oakood Place

Buffalo, New York 14214

*Claremont College

The Honnold Library

Periodicals Department

Claremont, California 91711

*H. Logan Cobb

Dept. of Foreign Lang.

Eastern Illinois University

Chatleston, Illinois 61920

Libraries

Serials Record Division

University of Notthern Colorado

Greeley, Colorado 80631

* Ferdinand V. Contino

92 Dennis Street

Manhasset, N. Y. 11030

*Dr. Marvin D'Lugo

Clark University

Dept. of Modern Langs.

950 Main Street

Worcester, Mass. 01610

*Alyce G. DeKuehne

Dept. of Romance \& Germanic Langs.

College of Liberal Arts

Wayne State Univ.

Detroit, Mich. 48202

*Dra. Angela B. Dellepiane

S10 E. 86th., Apt. 14-B

New York, N.Y. 10028

*Dr. Robert O. González-

Echevarría

Dept. of Rumance Studies

Cornell University

Ithaca, N. Y. 14850

*Dr. Albert de Ia Fuente

1002 E. 15th St.

Georgetown, Texas 78626

*Prof. Edward Dudley

Dept. of Hispanic Langs.

$\&$ Lits.

University of Pittsburgh

Pittsburgh, Pa. 15260 
*Prof. Julio Durán-Cerda University of Iowa Dept. of Romance Langs. Iowa City, Iowa 52240

*Prof. Robert L. Effland Spanish Department Marycrest College 1607 West 12th Street Davenport, Iowa 52804

*Dr. Peter G. Earle Logan Hall

University of $\mathrm{Pa}$.

Philadelphia, Pa. 19104

Eastern New Mexico Univ.

Library

Portales, New Mex. 88130

*Dr. John E. Englekirk

Dept. of Modern Langs.

University of California

Los Angeles, Calif. 96024

* Prof. Nalsy D. Ewing

2350 N. Mountain Ave.

Claremont, California 91711

-York S. Febres

2324 Orchard Place

New Brighton, Minn. 55112

* Oscar Fernández

Dept. of Spanish \& Port.

The University of Iowa

Iowa City, Iowa 52240

*Felina Ferragut

536 W. 113th St. No 56

New York, N. Y. 10025

*Joseph A. Feustle

Dept. of Foreign Langs.

Univ. of Toledo

Toledo, Ohio 43606

*Dr. Esperanza Figueroa. Amaral

Elmira College

Elmira, N. Y. 14901

*Dr. Nava E. Filer

Dept. of Foreign Langs.

Brooklyn College

Brooklyn, N. Y. 11210

*Prof. Maria T. Font

George Mason College of the

Univ. of Virginia

4400 University Drive

Fairfax, Virginia 22030

*Roslyn Frank

Dept. of Spanish \& Port.

University of Iowa

Iowa City, lowa 52240
Dr. Claudio Freixas

Calif. State Univ.

Humboldt

Spanish Dept.

Arcata, Calif. 95521

${ }^{*} \mathrm{M}$. Gallo

301 Oceano Ave., No. 2

Santa Bárbara, Calif. 93109

Dr. Víctor García

1150 Xenia Ave.

Yellow Springs, Ohio 45387

University of Georgia

Library

Athens, Georgia 30601

*Helmy F. Giacoman

Spanish Dept.

Adelphi University

Garden Citr. N.Y. 11530

- Robert M. Gleaves

Dept. of Foreign Langs.

University of N. Carolina

Charlotte, N. C. 28213

*Dr. Judith Goetzinger

14532114 th N. E.

Kirkland, Wash. 98033

*Dr. Stasys Gostautas

Wellesley College

P.O. BOX 36

Wellesley, Mass. 02181

*Miss lyonne R. Grove

Dept. of Foreing Langs.

Monmouth College

West Long Branch ${ }_{z}$ N.J. 07764

- Prof. A. A. Greene

202 Tuttle Rd.

San Antonio, Texas 78209

*Prof. William J. Grupp

Dept. of Span. \& Port.

University of Colorado

Boulder, Colo. 80302

Hiram College Library

P.O. Box 98

Hiram, Ohio 44234

*Julio L Hernández-Miyares

710 New Hyde Pk. Rd.

New Hyde Park, N. Y. 11523

Juan Amelia Hernández

Hood College Box 312

Frederick, Maryland 21701
* Mr. J. Herszenhorn

29-09 159th St.

Flushing, N. Y. 11358

* Carole Adele Holdworth R.608

831 South Harvey Ave.

Oak Park, Ill. 60304

*Tamara Holzapfel R-601

Dept. of Modern Langs.

University of New Mexico

Albuquerque, N.M. 87106

Library R-65s

Periodicals Section

University of Idaho

Moscow, Idaho 83843

Indiana University of $\mathbf{P a}$.

Library

Indiana, Penna, 15701

* James E. Irby R-657

Assoc. Prof. of Span. \& Pott.

Princeton Univ.

Princeton, N.J. 08540

*Dr. \& Mrs. T. B. Irving

Dept. of Romance Langs.

University of Tennessee

Knoxville, Tenn. 37916

State University of Iowa

Libraries

Serials Acquisition

Iowa City, Iowa 52240

* Didier T. Jaen R.553

Spanish Department

University of California

Davis, California 95616

José Olivio Jiménez R-554

215 W. 90th. St. Apt. 46

New York. N. Y. 10024

*Ernest A. Johnson R-602

195 West St.

Amherst, Mass, 01002

*Dr. Sonja Karsen

Dept. of Modern Languages

Skidmore College

Saratoga Springs, N.Y. 12866

*Dr. Ralph Kite

Dept. of Spanish \& Port.

University of Colorado

Boulder, Colo. 80302

*Linda B. Klein

Columbia University

Dept. of Spanish \& Port.

New York, N.Y. 10027 
*Robert B. Knox R-556

Dept. of Foreign Languages

Washington State University

Pullman, Wash. 99163

Phillip Koldewyn

Asst. Ptof. of Spanish

Claremont Men's College

Claremont, California 91717

*Prof. Anthony Lamb,

Chairman

Modern Langs. Dept.

Purdue Univ. Calumet

Campus

Hammond, Indiana 46323

*Myron E. Lichblau

Hall of Languages

Syracuse University

Syracuse, N.Y.

Cudahy Library

Loyola University

$6525 \mathrm{~N}$. Sheridan Rd.

Chicago, Ill. 60626

* Carlos Iozano

Dept. Modern Langs.

Calif. State College

Bakersfield, Calif. 93304.

*Leon F. Lyday

Dept. of Spanish, Ital \&

Port.

The Pennsylvania State Univ.

University Park, Pa. 16802

*Dr. Gerardo A. Luzuriaga

University of Calif., L.A.

Dept. of Spanish \& Port.

Los' Angeles, Calif. 90024

* Michnel Machlis

3340 S. Butte Ave., Apt. d5

Tempe, Arizona 85282

*Prof. Enrique MartínezLópez

Spanish \& Port. Dept.

University of California

Santa Barbara, Calif.

* Vera Maslow

315 W. 57th Street

The Towers

New York, N. Y. 10019

*Dr. Keith McDuffie,

Chairman

Dept. of Foreign Langs.

University of Montana

Missoula, Montana 59801

- Malcolm D. McLean

2555 Cockrell

Fort Worth, Texas 76109
* Prof. Robert G. Mead, Jr. Dept. of Romance Languages University of Conneticut

Storrs, Conn. 06268

*Thomas C. Meehan

Assist. Prof. of Spanish

Spanish Dept.

University of Illinois

Urbana, Illinois 61801

*W. L. Meinhardt

Foreign Langs. Dept.

Southern Illinois University

Carbondale, Illinois

*George Melnykovich

Box 286

Hiram, Ohio 44234

*Mrs. Giuseppe E. Mignone

350 West 51 st. St., Apt. 15F

New York, N:Y. 10019

*Esther P. Moceaga-González 516 N. 5th Street

DeKalb, Illinois 60115

*Prof. Casper J. Moresello

Dept. of Romance Languages

226 N. Grand Blvd.

Saint Louis University

St. Louis, Mo. 63103

*Robert J. Morris

Dept. of Classical $=$ Rom

Langs.

Texas Tech. University

Lubbock, Texas 79409

*Spanish Dept.

c/o Miss Leonor Andrade

Mount Mary College

Milwaukee, Wisconsin 53222

*Prof. Juan Moncayo

Modern Langs. Dept.

State Univ. College at Brockport

Brockport, N.Y. 11420

*Mr. William A. Morgan

Dept. of Foreign Langs.

Shippensburg State College

Shippensburg, Pa. 17257

${ }^{*}$ Luis Monguio

Dept. of Spanish \& Port.

University of California

Berkeley, California 94720

*Prof. Edwain C Munro

State Univ. of New York

1223 Western Ave.

Albany, N.Y.
*Frederic W. Murray

Dept. of Rom. Langs.

Northern Illinois University

Dekalb, Ill. 60115

* Marshall R. Näon

Director, Lang. \& Area Center

University of New Mexico

Albuquerque, New Mexico

*Prof. Arthur A. Natella

Dept. of Spanish \& Port.

University of Maryland

College Park, Maryland

20742

* Mrs. Zoila E. Nelken

37 Sixth Avenue

San Francisco, California

94118

- Graciela P. Nemes

6926 Pineway

Hyattsville, Maryland 20782

\section{CPO3478}

Nevada Southern

University Library

Periodicals

Las Vegas, Nevada 89109

*prof. Enrique Noble

Dept. of Modern Langs.

University of Missouri

St. Louis, Mo. 63121

* Otto Olivera

Dept. of Spanish Langs.

Tulane University

New Orleans, Ia. 70118

Library

Oregon State University

Corvallis, Oregon 97330

*Prof. Hextor H. Orjuela

Dept. of Spanish

Univ. of Calif. Irvine

Irvine, Calif. 92664

-Dr. Betty Tyree Osiek

Foreign Languages \&

Literatures

Southern Illinois University

Edwatdsville, Illinois 62025

*Mrs. Zaida Pagan

653 National Highway

LaVale, Md. 21502

* Pattee Library

Periodicals Section

Pennsylvania State University

University Park, Pa, 16802 
* Marta L. Pérez

6655 Marblow Drive

Jacksonville, Fla. 22211

* Prof. Lon Pearson

Dept. of Humanities

University of Missouri-Rolla

Rolla, Mo. 65401

*Dr. Esther Pereyra-Suarez

2244 Sierra Ventura Drive

Los Altos, Calif. 94022

*Prof. Andrea I. Pineda de Camps

1838 Russell

Dearborn, Mich. 48128

* Prof. Frank Pino, Jr.

Division of Foreign Langs.

University of Texas - San

Antonio

San Antonio, Texas 78284

-Helen Ponseti

110 Oak Ridge Av.

Iowa City, Jowa

Library

Princeton University

Princeton, N.J. 08540

- Prof. Luis Manuel Quesada Director, Florida States Canal

Zone Branch

P. O. Box 930

Albrook A. F. B. Panama Ca. nal

Zone 09825

*Miss Isis Quinteros

Modern Langs. Dept.

Saint Mary's College

Notre Dame, Indiana 46556

*Dr. Jorge Ramos

Route 1 Box 138

Pimento, Ind. 47866

- Susana Redondo de Feldman

Casa Hispánica

612 West 116th Street

New York, N. Y. 10027

*Dr. Richard M. Reeve

Dept. of Span. \& Port. Langs.

University of Calif.

Los Angeles, Calif. 90025

* Prof. José Rey-Barreau 1967 Goldsmith Lane E 14

Louisville, Kentucky 40218

- Dr. Rosario Rexach 301 E. 75th St., Apt. 9H

New York, N. Y. 10021
*University of Richmond

Library

Richmond, Virginia 23173

*Carlos Ripoll

Dept. of Spanish

Queens College

Flushing 67, New York

*Prof. James Willis Robb

George Washington

University

Dept. of Modern Iangs.

Washington $6, D$. C.

*William H. Roberts

Dept of Modern and Classical Langs.

Univ. of Mexico

Albuquerque, N. M. 87106

*Prof. José Rodríguez

Dept. of Foreign Languages

Illinois State University

Normal, Illinois 61761

*Prof. Doris Rolfe

Dept. of Foreign Langs. \&

Lits.

Norther Illinois Univ.

DeKalb, Illinois 60115

* Renato Rosaldo

\section{Chairman}

Dept. of Romance Langs.

University of Arizona

Tucson, Arizona 87521

*Dr. Mario E. Ruiz

Dept. of Romance Langs. \& Iits.

University of Cincinnati

Cincinnati, Ohio 45221

* María A. Salgado

Dept. of Romance Iangs.

The University of $\mathbf{N}$. Carolina

Chapel Hill, N. Carolina

Library, Serials Dept S.O.C.

San Fernando Valley State College

18111 Nordhoff Street

Northridge, Calif. 91324

*Dr. John F. Saunders

Dept. of Modern Langs.

Southwest Texas State Univ.

San Marcos, Texas 78666

*Prof. George O. Schanzer

230 Huxley Drive

Snyder, N. Y. 14226

* Prof. Peter J. Schoenbach

1621 Mt. Curve Ave.

Minneapolis, Minn. 55403
*Dr. Alan S. Schweitzer

Dept. of Modern Langs.

Texas A \& M University

College Station, Texas 77843

*Dr. Ivan A. Schulman

State University of N. Y.

Dept. of Hispanic Iangs.

Stony Brook, N. Y. 11790

*Dr. Federico Serra-Lima

13 Columbia Street

Oneonta, New York 13820

Lawrence A. Sharpe

P. O. Box 713

Universitv of North Carolina

Chapel Hill, N. C. 27514

* Prof. Rolf Sierra

228 Haight Street

Menlo, Calif. 94025

*Prof. Rodrigo Solera

Dept. of Foreign Langs.

Millersville State College

Millersville, $\mathrm{Pa} .17551$

*Mario T. Soria

4212 Pommel Place

West Des Moines, Iowa

50265

Ravmond D. Souza

1409 W. 22nd St.

Lawrence, Kans. 66044

*Edward D. Terry

P. O. Box 1422

University of Alabama

University, Alabama 35486

Mattin C. Taylor

Department of Modern Langs.

Univ. of Nebraska-Lincoln

Lincoln, Nebraska 68508

*E. Daymond Tumer

Graduate Office-UNCC

University Station

Charlotte, North Carolina 28213

*A. Valbuena Briones

203 Nottingham Road

Newark, Delaware 19711

*Dr. Andrés Valdespino

Dept. of Romance Langs.

Hunter College of the City

Univ. of New York

New York, N. Y. 10021

* Prof. Rosa E Valdés

217 Dresser Rd.

DeKalb, Illinois 60115 
*Mrs. Adriana H. Varela

13 Holly Drive

Hightstown, New Jersey 08520

*Ray Verzasconi

Dept. of Modern Languages

Oregon State University

Corvallis, Oregon 97331

*Francisco Villegas

406 Awixa Road

Ann Arbor, Michigan

Washington University

University Library

Saint Louis, Mo. 63130

Library

Wellesley College

Wellesley, Mass.

*Margarita Winikoff

9195 Collington Square

Allison Park, Pa. 15101

*Dr. Richard D. Woods

Dept. of Foreign Langs.

Trinity University

715 Stadium Drive

San Antonio, Texas 78212

*Hensley C. Woodbridge

1804 W. Freeman

Catbondale, Illinois 62901

*Dr. Donald A. Yates

537 Wells Hall

Michigan State University

E. Lansing, Michigan 48823

\section{AUSTRALIA}

National Library of Australia Canberra, A.C.T.

\section{CANADA}

*Keith Ellis

Dept. of Ital. \& Hispanic Studies

Iniversity of Toronto

Toronto 5 , Canadá

*Prof. Robert Glickman

Dept. of Ital. \& Hisp. Studies

University of Toronto

Toronto, Canada

*Prof. Richard L. Jackson

Dent. of Spanish

Catleton University

Ottawa 1, Canada
* Prof. Ross F. Larson

Dept. of Spanish

Carleton University

Ottawa 1, Ontario Canada

*Prof. Martha Martínez

235 Somerset St., W. Apt.

120

Ottawa 4, Ont., Canada

*Dr. Erminio G. Neglia

Dept. of Ital. \& Hisp. Stud.

University of Toronto

Toronto, Canada (5)

*Prof. Georges A. Parent

Depto. de Estudios Hispánicos

Université Laval

Quebec 10, Canadá

*Luis Pérez

University of Saskatchewan

Department of Spanish

Saskatoon, Canada

*Mrs. Josephine Tessier

59 Ruskin Avenue

Ottawa, Ont. Canada.

*Prof. Serge Zaitzeff

The University of Calgary

Romance Studies Department

Calgary Alberta, Canada

\section{CENTRAL AMERICA}

* Anelia W. de De León Regil 9a Calle 2-71 Zona 1

Guatemala, Guatemala, C. A

\section{ENGLAND}

*prof. G. W. Ribbans

School of Hisp. Studies

The University

P. O Box 147

Liverpool, England

\section{FRANCE}

*Prof. Claude Henri Freches

Directeur du Dept. D'Etudes

Port. \& Brés.-Univ. d'Aix-

Fac. des Let. et Sci HUM.

d'Aix en Provence

Marseille, France
ITALY

*Giuseppe Bellini

Via Simóne D'Orsenigo,

No. 5

20135 Milano, Italy

MEXICO

Prof. Alberto Gutiérrez A.

Instituto de Ciencias y Artes

de Chiapas

Tuxtla Gutiérrez, México

PERU

*Wolfgang A. Luchting

c/o García Btyce

Avenida Alvarez

Calderón 530-4

San Isidro

Lima, Perá

\section{PUERTO RICO}

* Mrs. Elena Ayala

Sec. Ejec. Ateneo Puert.

Darién 405, Villa Borinquen

Rio Piedras, P. R. 00920

*Edra Coll

Romaguera 185

Mayaguez, Puerto Rico

00708

Prof. José Ferrer-Canales

Box 22901

Univ. of Puerto Rico

Rio Piedras, P.R.

*Angel Luis Morales

Universidad de Puerto Rico

Dept de Estudios Hispánicas

Rio Piedras, Puerto Rico 00911

* Jaime Alberto Rosas-Rivera Dept. of Hispanic Studies

University of Puerto Rico

Mayaguez, Puerto Rico

\section{VENEZUELA}

* Carmelo Salvatierra

Universidad Católica Andrés Bello

Esquina Jesuitas, 37-Apt. 422

Caracas, Venezuela

- Patrón $-\$ 15.00$ more. 
